\title{
SOME CONSIDERATIONS REGARDING THE USE OF VAT PHOTOPOLYMERISATION TECHNIQUE IN PROSTHODONTICS
}

\author{
Edgar Moraru ${ }^{1}$, Octavian Dontu², Alina Spanu ${ }^{3}$, Delia Prisecaru ${ }^{4}$, Carmen Draghici ${ }^{5}$ \\ 1,2,3,4,5 University Politehnica of Bucharest \\ eddymilan91@yahoo.com,octavdontu@gmail.com,spanualina@yahoo.com \\ delia.prisecaru@hotmail.com, carmen.draghici3@gmail.com
}

\begin{abstract}
The article presents some aspects regarding the use of additive technologies that use liquid raw materials in prosthetic dentistry. The modern stages of obtaining 3D dental configuration will also be highlighted. In order to realize the customised dental models, it was used additive methods with photoreactive polymers, and namely SLA (stereolithography) and DLP (Digital Light Processing) methods. The models resulting from these advanced computerised techniques were investigated, drawing conclusions regarding the brought benefits of additive manufacturing methods in restorative dentistry and other medical applications.
\end{abstract}

Keywords: Additive Technologies, Prosthetic Dentistry, Vat Photopolymerisation.

\section{Introduction}

Conventional methods for realization of dental prostheses as casting, waxing, and lengthy refining are a thing of the past.

Numerous manual operations require a lot of time, high qualifications and as a result do not guarantee the necessary accuracy. Unlike subtractive production methods, additive technologies have practically no restrictions on the complexity of manufactured products, which makes it possible to produce monolithic objects with a complex internal structure.

High precision, measured in microns, is the best suited to the high requirements of dental prosthetics. Modern 3D printers allow to perform several tasks: from the manufacture of models and casting moulds to the production of finished metal prostheses and crowns with remarkable features [1].

In this paper, the additive technologies that use the raw material in liquid state (photoreactive polymers), which solidify under the action of ultraviolet light, were used. Liquid consolidation was achieved by two methods: SLA (stereolithography) and DLP (digital light processing).

SLA method is based on laser irradiation of liquid photopolymer resin to create solid physical models which is built layer by layer [2] [3]. Each layer is scanned by a laser according to the data embedded in a three-dimensional digital model.

Laser irradiation leads to the polymerization (i.e. solidification) of the material at the points of contact with the laser beam.

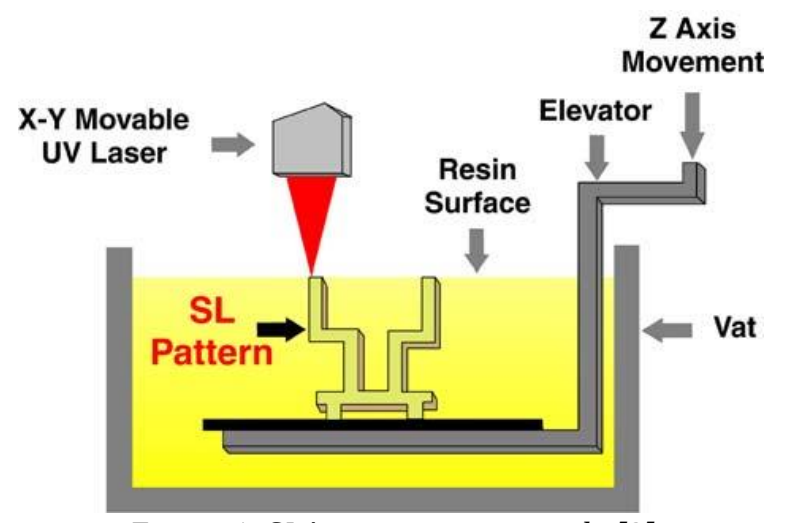

Figure 1: SLA operation principle [2]

An alternative method uses digital special projectors (DLP) to reduce the cost of devices. Unlike laser systems that scan the surface of a material with one or more laser heads, DLP printers project an image of a whole layer before the polymer resin hardens, after which a new layer of material is applied and an image of a new layer of a digital model is projected.

The main advantage of these systems can be considered high print accuracy. Existing technology allows to apply layers with a thickness of 15 microns, which is several times less than the thickness of a human hair.

The manufacturing accuracy is high enough for use in the manufacture of dental prostheses prototypes and jewellery.

Vat photopolymerisation methods allow to create parts of high complexity, but often has a high cost due to the relatively high price of consumables [1]. 


\section{Material and Methods}

The first step for the realization of some prosthetic elements is to obtain the three-dimensional model of interest. It can be obtained directly from the patient's oral cavity by scanning, this method being used also in this article. Intraoral scanning is becoming in modern conditions the standard of dental care. It replaced the traditional with taking impressions, significantly facilitating the process of restoration and prosthetics. Such scanning involves the use of a miniature sensor that transmits the image obtained by scanning the oral cavity to the screen of a computer connected to it. Thus, a threedimensional digital impression of the teeth is obtained.

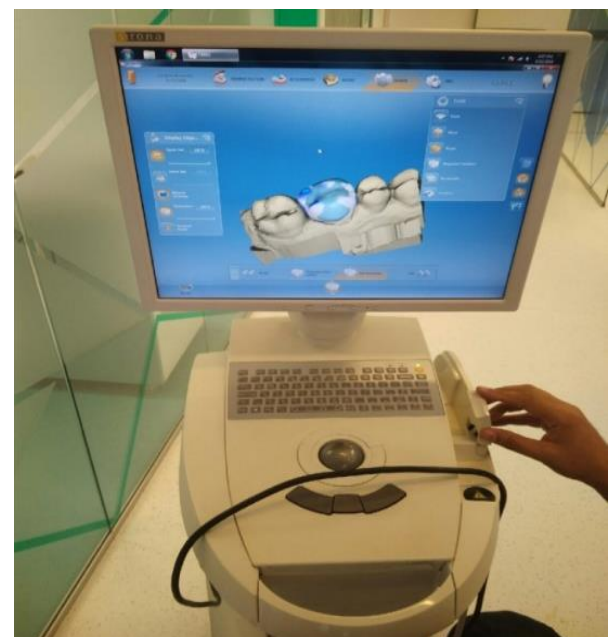

Figure 2: Result of intraoral scanning [4]

The following advantages of this scanning method can be distinguished: comfort of the patients; scanning speed (diagnosis takes about two minutes); the quality of the obtained 3D model (high-precision three-dimensional image allows the procedure of tooth restoration without the slightest error); detailing of photo and video images of the oral cavity in general and individual teeth in particular (resolution allows you to increase the studied area up to 100 times); qualitatively improves the treatment process and helps to obtain the most optimal results.

Statistics show that the use of intraoral scanning significantly reduces the percentage of revision after orthopedic and orthodontic treatment and improves its quality.

After inserting the scanner in the area of interest, the process of making the images will start automatically.

With this scanner, precise three-dimensional images in natural color are obtained, which simplifies the orientation in the dental cavity.

All previous metal, polymer or composite structures or prosthetic materials can be easily distinguished. An example of a scan result displayed directly on the computing unit is shown in Figure 2.

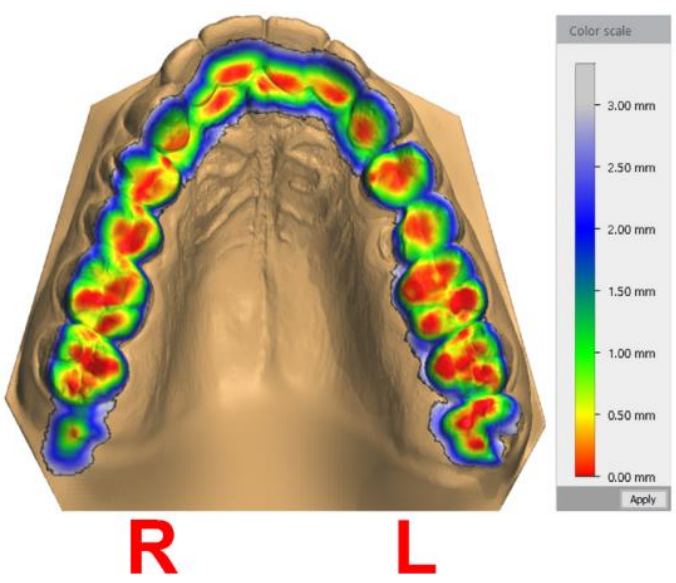

Figure 3: Generated occlusiogram

The scan results generate more information about the dental situation, including the occlusal data (Figure 3). An occlusiogram is used to determine the degree of tooth mobility, occlusal contacts, or periodontal tissue endurance.

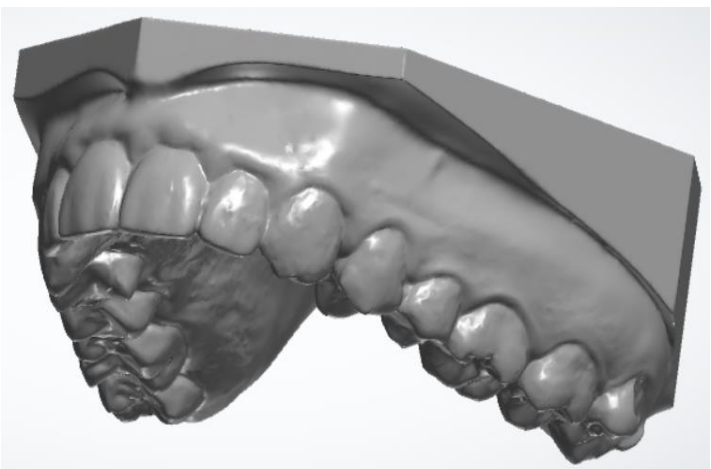

Figure 4: Scanning result of maxillary dental arcade

The intraoral scan was performed in a specialized centre of dental volumetric tomography [5], and the result of the intraoral scan for the maxillary arcade is shown in figure 4 . Based on the scans performed and obtained, dental models were made using the stereolithography (SLA) and DLP technologies, the execution steps of which are presented in the next chapter. The raw material for this technique is a liquid-state photopolymer with the property of solidifying under the action of ultraviolet light. In the case of SLA technology, a special dental resin was used, and in the case of DLP technology, Wanhao Standard resin was used, being an original polymer from the printer's manufacturers [6]. Two types of shades were used: white and black.

The polymer hardens under the action of a wavelength of $405 \mathrm{~nm}$ in about 10 seconds.

With a density of approximately $1.12 \mathrm{~g} / \mathrm{cm} 3$, this resin is one of the most used in the domain with very good mechanical properties. 


\section{Experimental}

Figure 5 shows the result of the three-dimensional printing of the complete dental model based on the intraoral scan [5]. The method used was stereolithography [3].

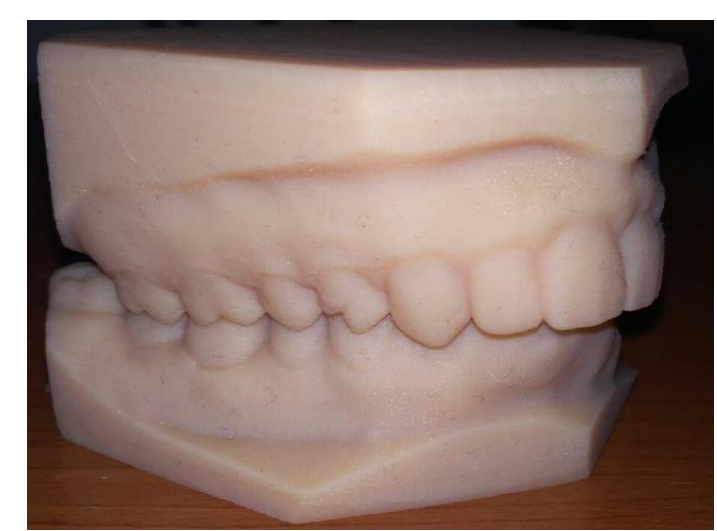

Figure 5: Maxillary and mandibular dental arcades realized by stereolitography

From the complete scan of an arcade, there is the possibility of separating certain pairs of teeth depending on the dental structures concerned and their applications. The upper incisors were chosen and separated from the maxillary arcade threedimensional model and executed using DLP technology. With this technology, various dental prototypes have been developed, which can serve as a model for future dental restorations. The piece is built on a horizontal platform immersed in the liquid polymer. The consolidation of the polymer is obtained by the photopolymerization realized at the impact of a light ray with the upper surface of the liquid.

The main element of DLP technology is the special device consisting from a matrix of micromirrors used for fast spatial light modulation. Each individual micro-mirror projects pixels from the cross-section of the three-dimensional model. Under the action of ultraviolet light, the photoreactive liquid (UV sensitive) solidifies in successive layers. Because the entire cross-section is designed in a single exposure, the rate of construction of a layer is constant, regardless of the complexity of the geometry [7] [8]

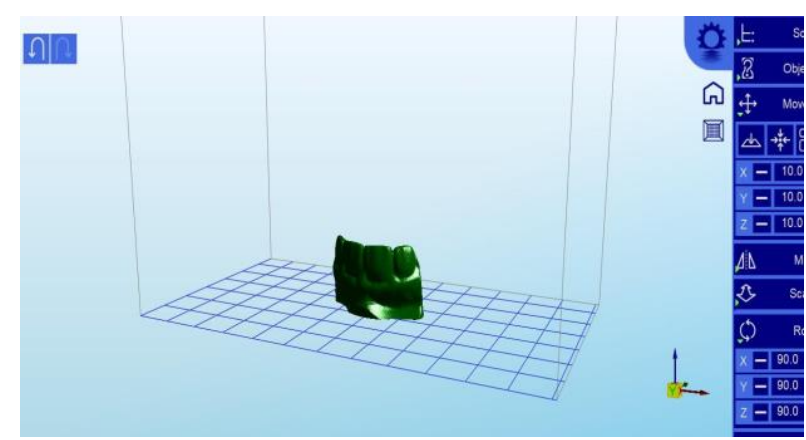

Figure 6. Creation Workshop software
The "Creation Workshop" software of the equipment was used [7]. This program allows the visualization and positioning of the workpiece, as well as the other useful operations. The program interface is presented in the Figure 6. The file is uploaded to the printer software and modelled. A very important step is the realization of the sacrificial layer of the structure. In this case there is a flat seating surface and it is not necessary to generate a sacrificial layer or support structure. From the program the working platform is immersed in the photopolymer liquid, the slicing operation is performed, after which the process can start, the program showing the estimated manufacturing time.

Also, the program can generate a simulation of the successive layers. The layer thickness for the DLP dental model was 50 microns, but also other layer thicknesses can be chosen from the software [8].

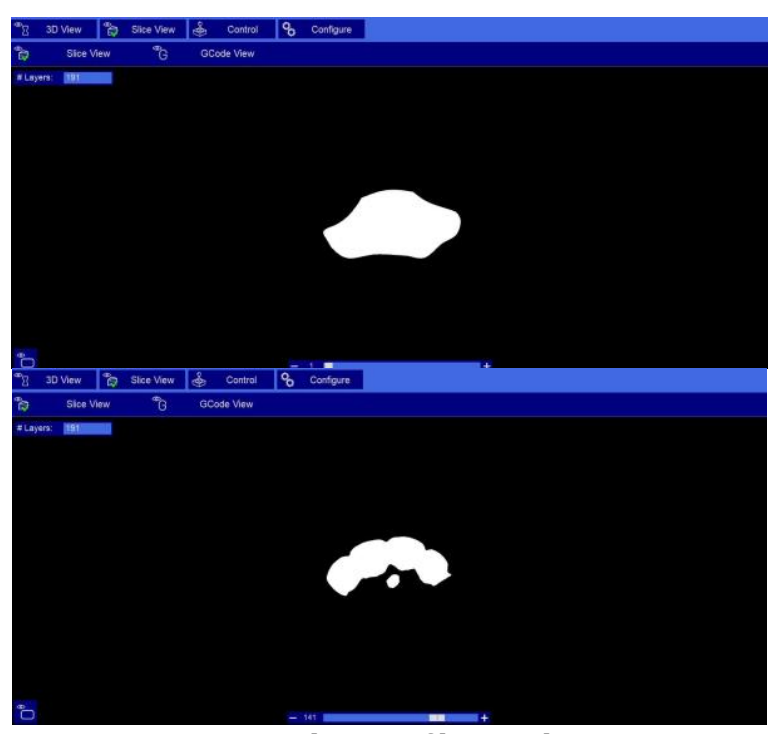

Figure 7: Simulation of layers deposition

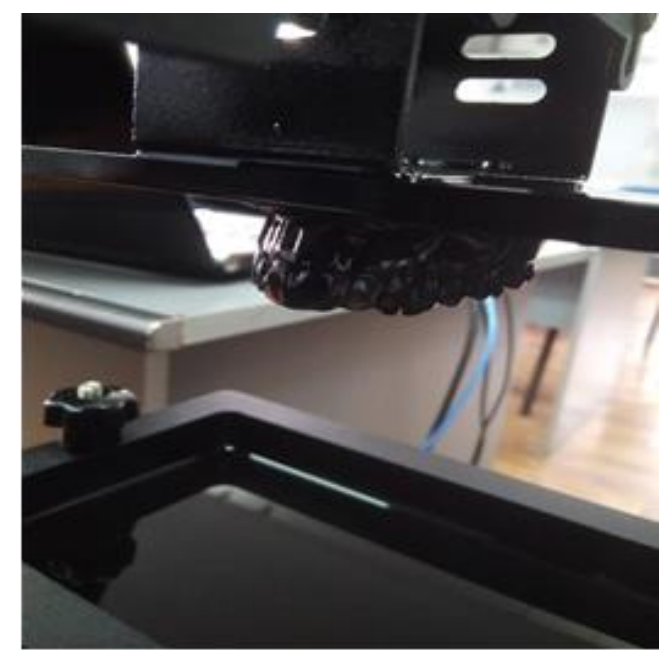

Figure 8: Dental models on the work platform [9]

To finish the photopolymerization process, the resulted structure is cleaned with technical alcohol solution and immersed for several hours in water. It is recommended to avoid contact with the sun's rays 
until the photopolymerization process is completed. Figure 8 shows the dental prosthetic model for the maxillary arcade realized by DLP technology, having a special appearance.

While thermoplastic extrusion technology produces a mechanical bond between layers, DLP technology creates a chemical bond by bonding photopolymers to layers, leading to very dense parts, the bond is water and air tight, and the resistance is not influenced by the orientation of the 3D model on the work platform. These dental prototypes obtained by vat photopolymerisation technology can be a landmark for the dentist for future prosthetic restoration.

Figure 9 shows a model made separately with the upper incisor teeth obtained from complete scanning of the maxillary. The dental structures generated by these two additive methods that use liquid raw material have a noteworthy aspect which can bring considerable benefits in the dental prosthetics and orthodontics domains [10].

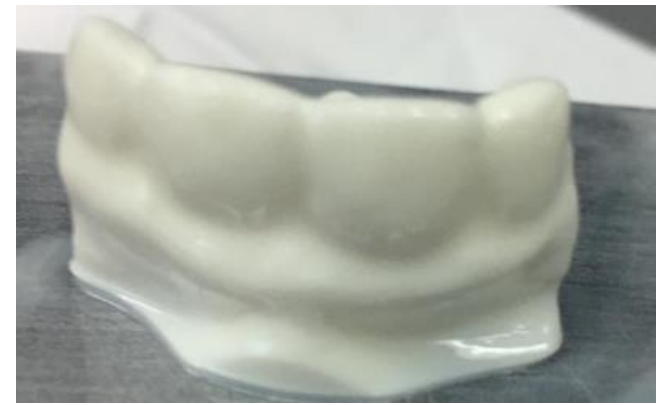

Figure 9: Resulted superior incisive model obtained from intraoral scanning realized by DLP technology

\section{Conclusions}

One of the most important characteristics in prosthodontics is the realization of restorations with high accuracy. The additive technology applicable to these tasks is simple, accurate, convenient to use, can significantly reduce the preparation and manufacturing time, and is also much more convenient for storing and working with data, ensuring $100 \%$ repeatability of dental products manufacturing (which is important in case of their breakage or loss). The resulting models match the shape and position in the oral cavity as much as possible. Fast digital production will allow you to quickly select the necessary parameters and geometry of future prostheses, and most importantly, exactly with the presentation of the surgeon and patient expectations. Scanning, file processing and printing are just three automated operations in the technological process, and a highquality result is obtained. Stereolithographic printers (SLA and DLP) allow the printing of master models for casting prostheses and crowns, eliminating the need for several intermediate steps. Diagnostic models of teeth printed on the basis of scanned individual impressions or obtained directly from the oral cavity using digital technologies, are necessary for accurate measurements and selection of elements of medical equipment. Also, possible applications can be the surgical templates and the preparation of the medical team for the complex surgical interventions. Based on the printed models, dentists get a clear picture of the affected area and can work out the intricacies of upcoming complex surgical operations. Future studies target the use of new special dental resins for DLP technology with improved properties and aspect.

\section{Acknowledgements}

This work has been funded by the European Social Fund from the Sectoral Operational Programme Human Capital 2014-2020, through the Financial Agreement with the title "Scholarships for entrepreneurial education among doctoral students and postdoctoral researchers (Be Antreprenor!)", Contract no. 51680/09.07.2019 - SMIS code: 124539.

\section{References}

[1] https://www.solver.ru/publications/publicatio ns.asp?id=2

[2] G.Carella, U.Galietti, D.Modugno, On the Feasibility of Thermoelastic Stress Analysis on Rapid Prototyping Models, Applied Mechanics and Materials 3-4:355-362

[3] D.Besnea, G.Ionascu, E.Manea, L.Bogatu, Development of a microstereophotolithography system, Romanian Review Precision Mechanics, Optics and Mechatronics, Iss.43, pp.126-130 (2013).

[4] Clinica dentara Trident, https://clinicatrident.ro/

[5] F.M. Medident - Radiologie si tomografie dentara, https://fmmedident.ro/

[6] Wanhao Duplicator 7 printer

[7] P.Berce, N.Balc, C.Caizar, R.Pacurar, A. S. Radu, S.Bratean, I.Fodorean, Tehnologii de fabricatie prin adaugare de material si aplicatiile lor, Editura Academiei Romane, Bucuresti, 2014.

[8] E.Moraru, D.Besnea, O.Dontu, G.I.Gheorghe, V.Constantin, Applications of Additive Technologies in Realization of Customized Dental Prostheses, International Journal of Mechatronics and Applied Mechanics, Iss.3, pp.66-71 (2018).

[9] E.Moraru, D.Besnea, A.Spanu, V.Constantin, Experimental research on some modern technologies and materials applied in prosthetic dentistry, Journal of Engineering Sciences and Innovation, Vol.3, Iss.4, pp.329-338 (2018).

[10] J.Anderson, J.Wealleance, J.Ray, Endodontic applications of 3D printing, Int Endod J. 2018 Sep; 51(9):1005-1018. 